\title{
VARIATIONS ON A THEME OF WIDDER
}

\author{
E. R. LOVE
}

These theorems, on functions having derivatives of all orders, are concerned with the equality of the four expressions appearing in (1) and (2) below. They arose out of a study of Widder's necessary and sufficient conditions for a function to be the Laplace-Stieltjes transform of a function of bounded variation. The purpose of the study was to obtain conditions under which a given function is the LaplaceStieltjes transform of a function of total variation less than 1 ; this question arose in work on dual integral equations. The results bring out an incidental symmetry, or duality, between the series and integral expressions in (1) and (2) that is surprisingly persistent. The proofs of Theorems 1-4 are given in the latter half of the paper, after the theorems have been stated and discussed. I am happy to record my thanks to Professor Widder and my appreciation of his interest in my work.

The original theme is:

THEOREM 0 (D. V. WIDDER). In order that a given function $f$ should be expressible by

$$
f(x)=\int_{0}^{\infty} e^{-x t} d \alpha(t) \quad \text { for all } x>0,
$$

where $\alpha$ is of bounded variation on $(0, \infty)$, it is necessary and sufficient that $f(x)$ have derivatives of all orders for all $x>0$ and that one of the following bounds be finite:

$$
\begin{gathered}
\sup _{x>0} \sum_{n=1}^{\infty}\left|f^{(n)}(x)\right| \frac{x^{n}}{n !}, \\
\sup _{n>0} \int_{0}^{\infty}\left|f^{(n)}(x)\right| \frac{x^{n-1}}{n-1 !} d x .
\end{gathered}
$$

See: D. V. Widder, The Laplace transform (Princeton Mathematical Series, Vol. 6, Princeton Univ. Press, Princeton, N. J., 1946), pp. 306-310, Theorems 12a and 13. The latter proves that finiteness of either of the above bounds entails that of the other. However, their actual equality is implicit in the proof. Further, Theorem 12b shows the possibility of replacing one of these bounds by a limit. These things are expressed a little more completely and generally in

Received by the editors June 29, 1963 and, in revised form, September 5, 1963. 
THEOREM 1. If $f(x)$ has derivatives of all orders in $x>a$ where $a \geqq 0$, and $p$ is a positive integer, then

$$
\sup _{x>a} \sum_{n=p}^{\infty}\left|f^{(n)}(x)\right| \frac{x^{n}}{n !}=\sup _{n \geq p} \int_{a}^{\infty}\left|f^{(n)}(x)\right| \frac{x^{n-1}}{(n-1) !} d x
$$

whether these bounds are finite or infinite. If they are finite, then the following limits exist and are equal to them:

$$
\lim _{x \rightarrow \infty} \sum_{n=p}^{\infty}\left|f^{(n)}(x)\right| \frac{x^{n}}{n !}=\lim _{n \rightarrow \infty} \int_{a}^{\infty}\left|f^{(n)}(x)\right| \frac{x^{n-1}}{(n-1) !} d x .
$$

Also the four equal expressions are independent of $a$ and $p$.

Widder also gives (p. 301) the following expression for the total variation of the determining function $\alpha$, supposed normalized at its discontinuities:

$$
V(\alpha ; 0, \infty)=\lim _{n \rightarrow \infty} \int_{0}^{\infty}\left|f^{(n)}(x)\right| \frac{x^{n-1}}{(n-1) !} d x+|f(\infty)| .
$$

Further information about this variation is implicit in his proofs. This, with additions which arise out of Theorem 1, gives

CoROLLARY 1. If $f$ is the Laplace transform of a function of bounded variation $\alpha$ normalized at its discontinuities, then the total variations of $\alpha(t)$ on $0<t<\infty$ and $0 \leqq t<\infty$ are, respectively:

$$
\begin{aligned}
V(\alpha ; 0+, \infty) & =\sup _{x>0} \sum_{n=1}^{\infty}\left|f^{(n)}(x)\right| \frac{x^{n}}{n !}=\sup _{n>0} \int_{0}^{\infty}\left|f^{(n)}(x)\right| \frac{x^{n-1}}{(n-1) !} d x \\
& =\lim _{x \rightarrow \infty} \sum_{n=1}^{\infty}\left|f^{(n)}(x)\right| \frac{x^{n}}{n !}=\lim _{n \rightarrow \infty} \int_{0}^{\infty}\left|f^{(n)}(x)\right| \frac{x^{n-1}}{(n-1) !} d x \\
V(\alpha ; 0, \infty) & =\lim _{x \rightarrow \infty} \sum_{n=0}^{\infty}\left|f^{(n)}(x)\right| \frac{x^{n}}{n !} .
\end{aligned}
$$

From here on the representation of $f$ by a Laplace transform, whether possible or not, is not needed at all; as in Theorem 1, and the proof of it here given, only existence of derivatives and their interrelations are used.

It is convenient to abbreviate the expressions in (1) and (2):

(3) $S_{p}(x)=\sum_{n=p}^{\infty}\left|f^{(n)}(x)\right| \frac{x^{n}}{n !}, \quad I_{a}(n)=\int_{a}^{\infty}\left|f^{(n)}(x)\right| \frac{x^{n-1}}{(n-1) !} d x$.

Equality of the bounds (1) and limits (2) under a simpler and ap- 
parently less restrictive condition than that of Theorem 1 is proved in Theorem 2. Whether it is possible or not to lighten this restriction still further, we shall see presently that some such condition cannot be entirely dispensed with.

THEOREM 2. If $f(x)$ has derivatives of all orders in $x>a$ where $a \geqq 0$, $p$ is a positive integer, and

$$
f^{(n)}(x) \frac{x^{n}}{n !}
$$

is bounded in $x>a, n \geqq p$, then the bounds (1) and the limits (2) are all four equal to one another, whether finite or infinite.

Further, $S_{p}(x)$ and $I_{a}(n)$ (see (3) above) are monotonic increasing in $x>a$ and in $n \geqq p$, respectively, for fixed $p$ and $a$.

The properties of the limits (2) stated in Theorems 1 and 2 can be obtained without proving the monotony of the expressions (3), perhaps even a little more simply. But this monotony seems to be worth proving-see Lemmas 2 and 4-for its own interest.

The equality of the bounds (1) and the limits (2) is, in Theorem 1, conditional on one of the bounds being finite, and, in Theorem 2 , on a relaxed version of this. One might ask whether existence and finiteness of one of the limits has the same implications; and whether the limits necessarily exist, finite or infinite, under more general conditions. These are the questions which led to Theorems 3 and 4; they were motivated to some extent by the likelihood that, in an application, the limits might be more easily found than the bounds.

THEOREM 3. If $f(x)$ has derivatives of all orders in $x>a$ where $a \geqq 0$, $p$ is a positive integer, and (using notation (3))

$$
\liminf _{n \rightarrow \infty} I_{a}(n)<\infty \text {, }
$$

then

$$
\lim _{x \rightarrow \infty} S_{p}(x)=\sup _{x>a} S_{p}(x)=\sup _{n \geq p} I_{a}(n) .
$$

If these are finite they are also equal to $\lim _{n \rightarrow \infty} I_{a}(n)$.

This finiteness hypothesis may be replaced by:

$$
f^{(n)}(x) \rightarrow 0 \text { as } x \rightarrow \infty, \text { for each } n \geqq p .
$$

This theorem has almost a dual with respect to the expressions (3), namely Theorem 4. The alternative "finiteness hypothesis" (10) in Theorem 4 is a little less simple than (7). 
THEOREM 4. If $f(x)$ has derivatives of all orders in $x>a$ where $a \geqq 0$, $p$ is a positive integer, and (using notation (3))

$$
\liminf _{x \rightarrow \infty} S_{p}(x)<\infty
$$

then

$$
\lim _{n \rightarrow \infty} I_{a}(n)=\sup _{n \geq p} I_{a}(n)=\sup _{x>a} S_{p}(x) .
$$

If these are finite they are also equal to $\lim _{x \rightarrow \infty} S_{p}(x)$.

This finiteness hypothesis may be replaced by:

$$
f^{(n)}(x) \text { is bounded in } c>x>a, \quad n \geqq p, \quad \text { for each } c>a .
$$

Combining these two theorems:

CoRollary 2. If both the lower limits in Theorems 3 and 4 are finite, that is, both (5) and (8) hold, then the bounds (1) and the limits (2) are all four equal to one another and finite, and $S_{p}(x)^{*}$ and $I_{a}(n)$ are monotonic increasing in $x>a$ and $n \geqq p$, respectively.

It is perhaps surprising that the hypothesis of Corollary 2 cannot be reduced to finiteness of only one of the lower limits. Whether $\lim$ inf $I_{a}(n)<\infty$ is sufficient I do not know; but certainly $\lim \inf S_{p}(x)<\infty$ is not sufficient, as the counterexample below shows. Further, the example shows that Theorem 1 cannot be simplified to the extent of omitting the finiteness hypothesis altogether; and that Theorem 2 is therefore worth stating.

COUNTEREXAMPLE. There is a function having derivatives of all orders for all $x \geqq 0$, indeed with all derivatives tending to zero as $x \rightarrow \infty$, for which (using notation (3))

$$
\underset{x \rightarrow \infty}{\liminf } S_{p}(x)=0 \text { and } \quad \limsup _{x \rightarrow \infty} S_{p}(x)=\infty
$$

for every positive integer $p$.

For such a function the two bounds (1), being equal by Theorem 1, are both infinite for every $a \geqq 0$ and every $p>0$.

For this function Lemma 2 shows that $I_{a}(n)$ is increasing, and Theorem 4 that $I_{a}(n) \rightarrow \infty$ as $n \rightarrow \infty$. Thus $I_{a}(n)$ is an increasing sequence tending to $\infty$ (for each $a \geqq 0$ ), in contrast with $S_{p}(x)$ which oscillates infinitely as $x \rightarrow \infty$ (for each $p>0$ ).

This example shows further that the last paragraph of Theorem 4 cannot be replaced by the simpler one of Theorem 3; and that the boundedness of (4) assumed in Theorem 2 cannot be relaxed so far as merely to suppose that $f^{(n)}(x) \rightarrow 0$ as $x \rightarrow \infty$. 
The example itself is the function $h$ given by

$$
h(x)=\sum_{r=1}^{\infty} \frac{1}{\sqrt{ } r} g(x-2 r),
$$

where $g$ is defined to be zero everywhere except in $(0,1)$ and

$$
g(x)=\exp \left(-\frac{1}{x^{2}}-\frac{1}{(1-x)^{2}}\right) \text { in } 0<x<1 .
$$

This function $g$ has derivatives of all orders everywhere, being the product of two well-known such functions; and $h$ has the same property since

$$
h(x)=0(2 r-1 \leqq x \leqq 2 r), \quad h(x)=\frac{1}{\sqrt{ } r} g(x-2 r)(2 r \leqq x \leqq 2 r+1) .
$$

The $n$th derivative of $g$ is bounded, since it is zero outside $(0,1)$ and continuous (being differentiable) on $[0,1]$. So the $n$th derivative of $h$ is $O(1 / \sqrt{ } x)$ as $x \rightarrow \infty$, and therefore tends to zero.

Since all derivatives of $h$ vanish at $x=2 r-1 / 2, S_{n}(x)$ has lower limit zero. Since $g$ is not polynomial in $(0,1), g^{(n)}\left(x_{n}\right) \neq 0$ for some $x_{n}$ in $(0,1)$, for each positive integer $n$. So

$$
\left|h^{(n)}\left(2 r+x_{n}\right)\right| \frac{\left(2 r+x_{n}\right)^{n}}{n !} \geqq \frac{\left|g^{(n)}\left(x_{n}\right)\right|}{\sqrt{ } r} \frac{(2 r)^{n}}{n !} \rightarrow \infty \quad \text { as } r \rightarrow \infty,
$$

whence, for each fixed $n$,

$$
\limsup _{x \rightarrow \infty} S_{n}(x) \geqq \limsup _{x \rightarrow \infty}\left|h^{(n)}(x)\right| \frac{x^{n}}{n !}=\infty .
$$

Proofs of the above four theorems occupy most of the rest of this paper.

Lемма 1. If $f(x)$ has derivatives of all orders in $x>a$ where $a \geqq 0$, $p$ and $q$ are positive integers, and

$$
f^{(n)}(x) \frac{x^{n}}{n !}
$$

is bounded in $x>a, q>n \geqq p$, then for each $b>a$

$$
\sum_{n=p}^{q-1}\left|f^{(n)}(b)\right| \frac{b^{n}}{n !} \leqq \int_{b}^{\infty}\left|f^{(q)}(x)\right| \frac{x^{q-1}}{(q-1) !} d x .
$$

If $p \leqq n<q$ and $c>b$, Taylor's theorem gives 


$$
\begin{aligned}
f^{(n)}(b) & =\sum_{r=0}^{q-n-1} f^{(n+r)}(c) \frac{(b-c)^{r}}{r !}+\int_{c}^{b} f^{(q)}(x) \frac{(b-x)^{q-n-1}}{(q-n-1) !} d x \\
& =\int_{\infty}^{b} f^{(q)}(x) \frac{(b-x)^{q-n-1}}{(q-n-1) !} d x, \quad \text { by making } c \rightarrow \infty .
\end{aligned}
$$

So

$$
\begin{aligned}
\sum_{n=p}^{q-1}\left|f^{(n)}(b)\right| \frac{b^{n}}{n !} & \leqq \sum_{n=p}^{q-1} \int_{b}^{\infty}\left|f^{(q)}(x)\right| \frac{(x-b)^{q-n-1}}{(q-n-1) !} \frac{b^{n}}{n !} d x \\
& =\int_{b}^{\infty}\left|f^{(q)}(x)\right| \sum_{n=p}^{q-1} \frac{(x-b)^{q-n-1}}{(q-n-1) !} \frac{b^{n}}{n !} d x \\
& \leqq \int_{b}^{\infty}\left|f^{(q)}(x)\right| \sum_{n=0}^{q-1} \frac{(x-b)^{q-1-n}}{(q-1-n) !} \frac{b^{n}}{n !} d x \\
& =\int_{b}^{\infty}\left|f^{(q)}(x)\right| \frac{x^{q-1}}{(q-1) !} d x
\end{aligned}
$$

LEMma 2. If $f(x)$ has derivatives of all orders in $x>a$, where $a \geqq 0$, $p$ and $q$ are positive integers, and for $p \leqq n<q$

$$
f^{(n)}(x) \rightarrow 0 \text { as } x \rightarrow \infty,
$$

then

$$
I_{a}(m)=\int_{a}^{\infty}\left|f^{(n)}(x)\right| \frac{x^{n-1}}{(n-1) !} d x
$$

is an increasing function of $n$ in $p \leqq n \leqq q$.

If $p \leqq n<q$ and $a<x<c$, $f^{(n)}(x)=f^{(n)}(c)-\int_{x}^{c} f^{(n+1)}(t) d t=-\int_{x}^{\infty} f^{(n+1)}(t) d t, \quad$ making $c \rightarrow \infty$.

So

$$
\begin{aligned}
\int_{a}^{\infty}\left|f^{(n)}(x)\right| \frac{x^{n-1}}{(n-1) !} d x & \leqq \int_{a}^{\infty} \frac{x^{n-1}}{(n-1) !} d x \int_{x}^{\infty}\left|f^{(n+1)}(t)\right| d t \\
& =\int_{a}^{\infty}\left|f^{(n+1)}(t)\right| d t \int_{a}^{t} \frac{x^{n-1}}{(n-1) !} d x \\
& \leqq \int_{a}^{\infty}\left|f^{(n+1)}(t)\right| \frac{t^{n}}{n !} d t, \quad \text { since } a \geqq 0 .
\end{aligned}
$$


LEMma 3. If $f(x)$ has derivatives of all orders in $b \geqq x>a$ where $a \geqq 0$, $p$ is a positive integer, and

$$
f^{(n)}(x) \frac{x^{n}}{n !}
$$

is bounded in $b>x>a, n \geqq p$, then for each positive integer $q \geqq p$

$$
\int_{a}^{b}\left|f^{(q)}(x)\right| \frac{x^{q-1}}{(q-1) !} d x \leqq \sum_{n=q}^{\infty}\left|f^{(n)}(b)\right| \frac{b^{n}}{n !} .
$$

If $\pm M$ are the bounds implied, and $a<x<b$, Taylor's theorem gives

$$
f^{(q)}(x)=\sum_{r=0}^{k-q} f^{(q+r)}(b) \frac{(x-b)^{r}}{r !}+R=\sum_{n=q}^{k} f^{(n)}(b) \frac{(x-b)^{n-q}}{(n-q) !}+R,
$$

where $k$ is any integer greater than $q$ and

$$
\begin{aligned}
R & =\int_{b}^{x} f^{(k+1)}(t) \frac{(x-t)^{k-q}}{(k-q) !} d t \\
|R| & \leqq \int_{x}^{b}\left|f^{(k+1)}(t)\right| \frac{(t-x)^{k-q}}{(k-q) !} d t \\
& \leqq \int_{x}^{b} M \frac{(k+1) !}{t^{k+1}} \frac{(t-x)^{k-q}}{(k-q) !} d t \\
& =M \frac{(k+1) !}{(k-q) !} \int_{x}^{b}\left(1-\frac{x}{t}\right)^{k-q} \frac{1}{t^{q+1}} d t \\
& \leqq M \frac{(k+1) !}{(k-q) !}\left(1-\frac{x}{b}\right)^{k-q} \int_{x}^{b} \frac{1}{t^{a+1}} d t \\
& \leqq \frac{M}{q x^{q}}(k+1)^{a+1}\left(1-\frac{x}{b}\right)^{k-q} \rightarrow 0 \text { as } k \rightarrow \infty .
\end{aligned}
$$

Thus the Taylor expansion

$$
f^{(q)}(x)=\sum_{n=q}^{\infty} f^{(n)}(b) \frac{(x-b)^{n-q}}{(n-q) !}
$$

holds in $a<x \leqq b$; and so 


$$
\begin{aligned}
\int_{a}^{b}\left|f^{(q)}(x)\right| \frac{x^{q-1}}{(q-1) !} d x & \leqq \int_{a}^{b} \sum_{n=q}^{\infty}\left|f^{(n)}(b)\right| \frac{(b-x)^{n-q}}{(n-q) !} \frac{x^{q-1}}{(q-1) !} d x \\
& =\sum_{n=q}^{\infty} \frac{\left|f^{(n)}(b)\right|}{(n-q) !(q-1) !} \int_{a}^{b}(b-x)^{n-q} x^{q-1} d x \\
& \leqq \sum_{n=q}^{\infty} \frac{\left|f^{(n)}(b)\right|}{(n-q) !(q-1) !} \int_{0}^{b}(b-x)^{n-q} x^{q-1} d x \\
& =\sum_{n=q}^{\infty}\left|f^{(n)}(b)\right| \frac{b^{n}}{n !}, \quad \text { as stated. }
\end{aligned}
$$

LEмma 4. If $f(x)$ has derivatives of all orders in $b \geqq x>a$ where $a \geqq 0$, $p$ is a positive integer, and $f^{(n)}(x) x^{n} / n$ ! is bounded in $b>x>a, n \geqq p$, then

$$
S_{p}(x)=\sum_{n=p}^{\infty}\left|f^{(n)}(x)\right| \frac{x^{n}}{n !}
$$

is an increasing function of $x$ in $a<x \leqq b$.

As in the proof of Lemma 3 , if $a<x<b$ and $q \geqq p$,

$$
f^{(a)}(x)=\sum_{n=q}^{\infty} f^{(n)}(b) \frac{(x-b)^{n-q}}{(n-q) !} .
$$

So

$$
\begin{aligned}
\sum_{q=p}^{\infty}\left|f^{(q)}(x)\right| \frac{x^{q}}{q !} & \leqq \sum_{q=p}^{\infty} \frac{x^{q}}{q !} \sum_{n=q}^{\infty}\left|f^{(n)}(b)\right| \frac{(b-x)^{n-q}}{(n-q) !} \\
& =\sum_{n=p}^{\infty}\left|f^{(n)}(b)\right| \sum_{q=p}^{n} \frac{(b-x)^{n-q}}{(n-q) !} \frac{x^{q}}{q !} \\
& \leqq \sum_{n=p}^{\infty}\left|f^{(n)}(b)\right| \frac{b^{n}}{n !}, \quad \text { since } p>0 .
\end{aligned}
$$

In this argument $b$ can be replaced by any smaller number exceeding $x$, and this gives the stated result.

Lemma 5. If $f(x)$ has derivatives of all orders in $x>a$ where $a \geqq 0$, $p$ and $q$ are positive integers, and

$$
\int_{a}^{\infty}\left|f^{(n)}(x)\right| \frac{x^{n-1}}{(n-1) !} d x<\infty \quad \text { for } q \geqq n \geqq p,
$$

then $f^{(n)}(x) x^{n} / n !$ is bounded in $x>a, q>n \geqq p$ (as needed for Lemma 1). 
Suppose $b>a, x>a$, and $q>n \geqq p$. Then

$$
\int_{b}^{x} f^{(n+1)}(t) \frac{t^{n}}{n !} d t=f^{(n)}(x) \frac{x^{n}}{n !}-f^{(n)}(b) \frac{b^{n}}{n !}-\int_{b}^{x} f^{(n)}(t) \frac{t^{n-1}}{(n-1) !} d t,
$$

and so, whether $x>b$ or $x<b$,

$$
\begin{aligned}
\left|f^{(n)}(x)\right| \frac{x^{n}}{n !} \leqq & \left|f^{(n)}(b)\right| \frac{b^{n}}{n !}+\int_{a}^{\infty}\left|f^{(n+1)}(t)\right| \frac{t^{n}}{n !} d t \\
& +\int_{a}^{\infty}\left|f^{(n)}(t)\right| \frac{t^{n-1}}{(n-1) !} d t .
\end{aligned}
$$

The left-hand side is bounded, for $x>a$ and $q>n \geqq p$, by the greatest of the finite set of values of the right-hand side for $q>n \geqq p$.

Proof of TheOREM 1. Whether finite or infinite, write

$$
L=\sup _{x>a} S_{p}(x), \quad M=\sup _{x \geq 0} I_{a}(n) ;
$$

where, as in (3),

$$
S_{p}(x)=\sum_{n=p}^{\infty}\left|f^{(n)}(x)\right| \frac{x^{n}}{n !}, \quad I_{a}(n)=\int_{a}^{\infty}\left|f^{(n)}(x)\right| \frac{x^{n-1}}{(n-1) !} d x .
$$

(i) If $L<\infty, f^{(n)}(x) x^{n} / n$ ! is bounded in $x>a, n \geqq p$, so Lemma 3 gives

$$
\int_{a}^{b}\left|f^{(q)}(x)\right| \frac{x^{q-1}}{(q-1) !} d x \leqq S_{q}(b) \leqq S_{p}(b) \leqq L
$$

for any $b>a$ and any integer $q \geqq p$; thus

$$
\int_{a}^{\infty}\left|f^{(q)}(x)\right| \frac{x^{q-1}}{(q-1) !} d x \leqq L, \text { and so } M \leqq L .
$$

(ii) If $M<\infty$ instead, and $q$ is any integer greater than $p$, Lemma 5 shows that $f^{(n)}(x) x^{n} / n$ ! is bounded in $x>a, q>n \geqq p$. So Lemma 1 gives

$$
\sum_{n=p}^{q-1}\left|f^{(n)}(b)\right| \frac{b^{n}}{n !} \leqq I_{b}(q) \leqq I_{a}(q) \leqq M
$$

for any $b>a$; whence

$$
\sum_{n=p}^{\infty}\left|f^{(n)}(b)\right| \frac{b^{n}}{n !} \leqq M, \text { and so } L \leqq M .
$$


Together (i) and (ii) show that if either $L$ or $M$ is finite then so is the other and they are equal. This proves (1).

Now suppose $L$ and $M$ are finite. By definition of $L, f^{(n)}(x) x^{n} / n !$ is bounded in $x>a, n \geqq p$. By Lemma $4, S_{p}(x)$ is an increasing function of $x$ in $x>a$; so it converges to its upper bound $L$ as $x \rightarrow \infty$. By Lemma 2, $I_{a}(n)$ is an increasing function of $n$ in $n \geqq p$; so it converges to its upper bound $M$ as $n \rightarrow \infty$. These remarks establish (2).

Proof of Theorem 2. By Theorem $1, L=M$ whether finite or infinite. Lemmas 4 and 2 used as in the preceding paragraph again show that $S_{p}(x)$ is increasing with limit $L$ as $x \rightarrow \infty$, and $I_{a}(n)$ is increasing with limit $M$ as $n \rightarrow \infty$, whether $L$ and $M$ are finite or infinite. This proves Theorem 2 .

We now prepare to prove Theorems 3 and 4. The next two lemmas generalize Lemmas 1 and 3, omitting the hypothesis of boundedness of $f^{(n)}(x) x^{n} / n$ !

Lemma 6. If $f(x)$ has derivatives of all orders in $c \geqq x \geqq b$ where $b>0$, $p$ and $q$ are positive integers and $q>p$, then

$$
\sum_{n=p}^{q-1}\left|f^{(n)}(b)\right| \frac{b^{n}}{n !} \leqq \sum_{n=p}^{q-1}\left|f^{(n)}(c)\right| \frac{c^{n}}{n !}+\int_{b}^{c}\left|f^{(q)}(x)\right| \frac{x^{q-1}}{(q-1) !} d x .
$$

Taylor's theorem gives, if $p \leqq n<q$,

$$
f^{(n)}(b)=\sum_{m=n}^{q-1} f^{(m)}(c) \frac{(b-c)^{m-n}}{(m-n) !}+\int_{c}^{b} f^{(q)}(x) \frac{(b-x)^{q-1-n}}{(q-1-n) !} d x,
$$

so that

$$
\begin{aligned}
& \sum_{n=p}^{q-1}\left|f^{(n)}(b)\right| \frac{b^{n}}{n !} \\
& \leqq \sum_{n=p}^{q-1} \sum_{m=n}^{q-1}\left|f^{(m)}(c)\right| \frac{(c-b)^{m-n}}{(m-n) !} \frac{b^{n}}{n !}+\sum_{n=p}^{q-1} \int_{b}^{c}\left|f^{(q)}(x)\right| \frac{(x-b)^{q-1-n}}{(q-1-n) !} \frac{b^{n}}{n !} d x \\
& =\sum_{m=p}^{q-1}\left|f^{(m)}(c)\right| \sum_{n=p}^{m} \frac{(c-b)^{m-n}}{(m-n) !} \frac{b^{n}}{n !}+\int_{b}^{c}\left|f^{(q)}(x)\right| \sum_{n=p}^{q-1} \frac{(x-b)^{q-1-n}}{(q-1-n) !} \frac{b^{n}}{n !} d x \\
& \leqq \sum_{m=p}^{q-1}\left|f^{(m)}(c)\right| \frac{c^{m}}{m !}+\int_{b}^{c}\left|f^{(q)}(x)\right| \frac{x^{q-1}}{(q-1) !} d x,
\end{aligned}
$$

the last step being to decrease the lower terminal $p$ of the $n$-summations to 0 to make complete binomial expansions. This proves the inequality. 
LEMmA 7. If $f(x)$ has derivatives of all orders in $b \geqq x>a$ where $a \geqq 0$, $q$ and $r$ are positive integers and $r>q$, then

$$
\int_{a}^{b}\left|f^{(q)}(x)\right| \frac{x^{q-1}}{(q-1) !} d x \leqq \int_{a}^{b}\left|f^{(r)}(x)\right| \frac{x^{r-1}}{(r-1) !} d x+\sum_{n=q}^{r-1}\left|f^{(n)}(b)\right| \frac{b^{n}}{n !} .
$$

Taylor's theorem gives, if $a<x<b$,

$$
f^{(q)}(x)=\sum_{n=q}^{-1} f^{(n)}(b) \frac{(x-b)^{n-q}}{(n-q) !}+\int_{b}^{x} f^{(r)}(t) \frac{(x-t)^{r-1-q}}{(r-1-q) !} d t,
$$

so that

$$
\begin{aligned}
\int_{a}^{b}\left|f^{(q)}(x)\right| & \frac{x^{q-1}}{(q-1) !} d x \\
& \leqq \int_{a}^{b} \sum_{n=q}^{r-1}\left|f^{(n)}(b)\right| \frac{(b-x)^{n-q}}{(n-q) !} \frac{x^{q-1}}{(q-1) !} d x \\
& \quad+\int_{a}^{b} d x \int_{x}^{b}\left|f^{(r)}(t)\right| \frac{(t-x)^{r-1-q}}{(r-1-q) !} \frac{x^{q-1}}{(q-1) !} d t \\
& =\sum_{n=q}^{r-1}\left|f^{(n)}(b)\right| \int_{a}^{b} \frac{(b-x)^{n-q}}{(n-q) !} \frac{x^{q-1}}{(q-1) !} d x \\
& \quad+\int_{a}^{b}\left|f^{(r)}(t)\right| d t \int_{a}^{t} \frac{(t-x)^{r-1-q}}{(r-1-q) !} \frac{x^{q-1}}{(q-1) !} d x \\
& \sum_{n=q}^{r-1}\left|f^{(n)}(b)\right| \frac{b^{n}}{n !}+\int_{a}^{b}\left|f^{(r)}(t)\right| \frac{t^{r-1}}{(r-1) !} d t
\end{aligned}
$$

the last step being to decrease the lower terminal $a$ of the $x$-integrations to 0 to make complete beta-functions. This proves the inequality.

Although Lemma 7 is not required, it has been included to exhibit the remarkable duality between the series and integral portions of the Taylor expansion. This duality shows in all the preceding work, but nowhere more completely than in the details of the statements and proofs of Lemmas 6 and 7.

Proof of Theorem 3. We may suppose that, in addition to (5),

$$
\liminf _{x \rightarrow \infty} S_{p}(x)<\infty,
$$

for otherwise $S_{p}(x) \rightarrow \infty$ as $x \rightarrow \infty$ and the conclusions stated are immediate, using Theorem 1 (1).

Lemma 6 gives, if $c>b>a$ and $q>p$, 


$$
\begin{aligned}
& \sum_{n=p}^{q-1}\left|f^{(n)}(b)\right| \frac{b^{n}}{n !} \leqq \sum_{n=p}^{\infty}\left|f^{(n)}(c)\right| \frac{c^{n}}{n !}+\int_{a}^{\infty}\left|f^{(q)}(x)\right| \frac{x^{q-1}}{(q-1) !} d x \\
& \sum_{n=p}^{\infty}\left|f^{(n)}(b)\right| \frac{b^{n}}{n !} \leqq \sum_{n=p}^{\infty}\left|f^{(n)}(c)\right| \frac{c^{n}}{n !}+\liminf _{a \rightarrow \infty} \int_{a}^{\infty}\left|f^{(q)}(x)\right| \frac{x^{q-1}}{(q-1) !} d x \\
& \sum_{n=p}^{\infty}\left|f^{(n)}(b)\right| \frac{b^{n}}{n !} \leqq \liminf _{c \rightarrow \infty} \sum_{n=p}^{\infty}\left|f^{(n)}(c)\right| \frac{c^{n}}{n !} \\
&+\liminf _{a \rightarrow \infty} \int_{a}^{\infty}\left|f^{(q)}(x)\right| \frac{x^{q-1}}{(q-1) !} d x \\
&= \liminf _{x \rightarrow \infty} S_{p}(x)+\liminf _{n \rightarrow \infty} I_{a}(n) .
\end{aligned}
$$

The left-hand side is thus bounded in $b>a$, by (5) and (11). So Theorem 1 applies, completing the proof of the first paragraph of the theorem.

If we assume only (5) and (7), Lemma 2 shows that $I_{a}(n)$ is increasing in $n \geqq p$. Thus its upper bound is equal to its limit as $n \rightarrow \infty$, and this is finite by hypothesis. So the conclusions again follow from Theorem 1.

The monotonic properties of $S_{p}(x)$ and $I_{a}(n)$ obtained in Theorem 2 do not necessarily hold in Theorem 3; at least this proof does not establish them in the case in which $S_{p}(x) \rightarrow \infty$ as $x \rightarrow \infty$.

Proof of Theorem 4. We may suppose that, in addition to (8),

$$
\liminf _{n \rightarrow \infty} I_{a}(n)<\infty,
$$

for otherwise $I_{a}(n) \rightarrow \infty$ as $n \rightarrow \infty$ and the conclusions stated are immediate, using Theorem 1 (1).

Lemma 6 now applies, just as in the proof of Theorem 3; and appeal to (8) and (12) establishes the first paragraph of Theorem 4.

Now assume only (8) and (10). Whenever $c>b>a$,

$$
\int_{b}^{c}\left|f^{(n)}(x)\right| \frac{x^{n-1}}{(n-1) !} d x=O\left(\frac{c^{n}}{n !}\right) \rightarrow 0 \text { as } n \rightarrow \infty .
$$

Making $q \rightarrow \infty$ in Lemma 6, this shows that

$$
\sum_{n=p}^{\infty}\left|f^{(n)}(b)\right| \frac{b^{n}}{n !} \leqq \sum_{n=p}^{\infty}\left|f^{(n)}(c)\right| \frac{c^{n}}{n !} .
$$

So now $S_{p}(x)$ is increasing in $x>a$; thus its upper bound is equal to its limit as $x \rightarrow \infty$, and this is finite by hypothesis. So the conclusions again follow from Theorem 1.

University of Melbourne, Victoria, Australia 